\title{
Tackling climate change with blockchain
}

Peter Howson*

School of Arts and Humanities, Nottingham Trent University, Nottingham NG11 8NS, UK

*Correspondence: peter.howson@ntu.ac.uk

Concern around the carbon footprint of Bitcoin is not holding back blockchain developers from leveraging the technology for action on climate change. Whilst blockchain is enabling individuals, companies and even cities to manage their carbon emissions, the social and environmental costs and benefits of doing so remain unclear.

This July saw the release of the University of Cambridge's Bitcoin Electricity Consumption Index (CBECI), an online tool providing real-time conjectures around the electricity requirements of the Bitcoin network. The prestige afforded towards Cambridge is likely to propel the CBECI model ahead of the popular Digiconomist tool, which released its Bitcoin Energy Consumption Index in 2017. Despite the apparent advancement, the increasingly complex modelling of Bitcoin's energy requirements is yet to provide any further clarity. The $\mathrm{CBECl}$ analysis, for example, suggests that total power consumption, at the time of writing this, falls within a range of between 21 Twh and 146 Twh. To put that in perspective, those figures equate to somewhere between the energy consumption of Yorkshire and Poland ${ }^{1}$. The network's energy requirements are as erratic as Bitcoin's price, depending as much on local weather events near remote Chinese hydroelectric plants, as the efficiency of energy-intensive servers that facilitate the Bitcoin blockchain ${ }^{2}$.

Bitcoin's computationally-demanding infrastructure enables digital payments to be validated via a decentralized, automated 'Proof of Work' (PoW) consensus protocol. For Bitcoin, the validation of transactions requires 'miners' using dedicated servers to solve hash puzzles in order to add valid entries to a shared database, and to secure new Bitcoins as a reward. The difficulty of these puzzles 
adjusts regularly to account for changes in connected computing power and to maintain approximately 10 minutes between the additions of each new block ${ }^{3}$. The likely carbon footprint of this process is significant, but the many unknowns are leading to wildly varying estimates. Mora et al. ${ }^{4}$ estimates that the computer processing power needed for the Bitcoin network alone could result in a global temperature rise of $2^{\circ} \mathrm{C}$ by 2050 . Others say such estimates are inflated, possibly by as much as $75 \%^{5}$, as miners increasingly flock to sources of cheap renewable energy, like hydropower and geothermal ${ }^{6,7}$. Due to this uncertainty, it is perhaps too early to abandon Bitcoin, or at least the potential of its underlying technology.

\section{Developing climate-smart blockchain platforms}

Central to Bitcoin, and all the other 'alt-coins' that have followed its ascendency, is blockchain technology. A blockchain is the system's distributed and immutable electronic database - a ledger of every transaction that has ever taken place on the network. Data is stored as cryptographically secured 'blocks', strung together in a 'chain'. While Bitcoin was the first application of blockchain, cryptocurrencies are just one of its many uses. Blockchain applications include government recordkeeping, tracking the flow of goods and services along supply chains, voting, and verifying the identity of citizens. Blockchain also has capabilities far beyond any ordinary database, because the technology uses algorithms to facilitate 'smart contracts'. Self-executing code provides secure mechanisms for electronic collaboration that do not rely upon a central authority to mediate between transacting parties. These parties, who might not necessarily trust each other, can trust the authenticity of information held in their shared databases ${ }^{8}$.

Although they all use the same general approach based on a peer-to-peer network, accommodating a permanent and secure ledger, each blockchain may use different consensus protocols for validating data ${ }^{9}$. Not all these blockchain validation protocols are especially energy intensive. The 'Proof of Stake' (PoS) protocol, as used by the DASH blockchain, and proposed for future iterations of Ethereum, requires less than $1 \%$ of the energy consumption needed for PoW ${ }^{10}$. 'Delegated Proof 
of Stake' (DPoS), used by the EOS blockchain, and NEO's 'delegated Byzantine Fault Tolerance' (dBFT) model, conduct community elections to grant validating power to stake-holding nodes. These alternative validation models tend to sacrifice certain security features and decentralised governance arrangements, but enable faster, more scalable, efficient and, possibly, more climatefriendly blockchain platforms.

\section{Carbon offsets on the blockchain}

There are many climate-conscious blockchain initiatives in various stages of development. SolarCoin, for example, uses a blockchain platform to incentivise solar energy producers by rewarding every MWh of electricity they produce with 1 free SolarCoin. This digital reward can be used as a medium of exchange, or converted to any other currency. Projects like Earth Dollar aim to link carbon credits (pollution permits that are issued for emissions avoided elsewhere) to blockchain tokens (representations of a particular asset or utility within the platform). Some initiatives are enabling automated smart-contract payment protocols, so that embodied carbon emissions from consumer purchases can be calculated and carbon credits purchased automatically. Infinite Earth's Veridium Labs, for example, a Hong Kong-based private company working in partnership with IBM, are connecting their payment system (VerdePay) with carbon credits produced from Infinite Earth's forest reserve in Rimba Raya, Central Kalimantan.

Ecosphere+ and Althelia, a natural-asset management company based in Luxemburg, are bringing their carbon credits to market using blockchain tokens. The carbon credits originate from conservation efforts in Peru's Cordillera Azul National Park. These credits are being provided to its strategic Maltese partner, Poseidon, whose blockchain platform allows consumers and retailers to track and offset their carbon footprints using Ocean tokens ${ }^{11}$. Poseidon has already partnered with Liverpool City Council and the London store of Ben \& Jerry's ice cream.

Another carbon offset initiative, EarthToken, proposes something similar to Poseidon and Veridium, while also claiming to issue blockchain tokens to local tree planters, incentivising conservation in 
Zimbabwe's Kariba forest. This claim has attracted criticism. In reality, no payments to forest communities in Zimbabwe has ever been made in exchange for tree planting efforts, using Earth Tokens or any other mechanism ${ }^{8}$.

\section{The social impacts of a blockchain climate fix}

The climate impacts of blockchain projects are global, as are their decentralised governance frameworks, but they are not placeless. The social impacts of each are centralised locally, often exacerbating structural inequalities. Instead of reaching local host communities, Poseidon's profits from crypto-carbon sales are used to repay loans from the projects' private investors. The project is neither financially compensating local people, nor directly incentivising any tree planting activities other than those that had already taken place ${ }^{12}$. Climate-conscious investors and consumers using Veridium's platform may also be better off donating money directly to ongoing tree planting initiatives. The Veridium project's tokens represent carbon that was sequestered in Rimba Raya's forest reserve several years ago. According to Enrici and Hubacek ${ }^{13}$, no financial compensation makes it to the local communities paying the highest costs for these enclosures.

Connecting carbon credits to cryptocurrencies is increasing market access ${ }^{8}$, but despite offering a theoretically 'trustworthy', and accessible means of carbon commodity exchange, cryptocurrencies are not currently capable of accurately representing the dynamic materiality of forests, nor the communities that make a livelihood from them. After 25 years of carbon-offsetting, the writing on the wall for these schemes is becoming clear. Even the UN architects of incentive-based schemes such as Reducing Emissions of Deforestation and Forest Degradation (REDD+), have recently concluded that the era of carbon trading is drawing to a close, stating that, "carbon offsets have been used by polluters as a free pass for inaction" ${ }^{14}$. Despite this, incentive-based approaches that merge blockchain with other disruptive technologies - machine learning Artificial Intelligence and the 'Internet of Things', for example - are enabling some instances of effective climate change mitigation, while also steering paths towards more socially equitable outcomes. Regen Network uses 
blockchain technology to monitor and verify environmental performance, share data and facilitate incentive payments to local land stewards. They propose to use automated remote sensors to generate reliable attestations about the change in health of any predefined geographical area. According to Regen, the core set of 'Change of State' protocols and remote sensing tools have been co-produced by working groups of blockchain application developers, ecologists, farmers and forest communities $^{15}$.

This technological response to tackling climate change is perhaps cause for optimism. Remaining overly fixated on the inefficiency of some cryptocurrencies is likely to encourage throwing the blockchain baby out with Bitcoin's bathwater. Crypto-projects like Regen's use validation protocols that do not require the energy intensive computational power of more established blockchain protocols. Regen proposes governance and consensus mechanisms which may promote greater participation from forest-dependent communities. By cutting out expensive intermediaries they may also have the potential to reconfigure global patterns of inequality, allowing communities in the Global South to access some of the financial benefits of a global green economy; benefits they have previously been denied ${ }^{16}$. This is important, because until the focus shifts towards achieving more equitable outcomes, not only will tackling climate change with blockchain technology lead to an oversimplification of socio-ecological complexity, it will reproduce the past failures of incentivebased mechanisms and other false solutions for climate crises.

\section{Full article here:}


1 [IEA] International Energy Agency. Global energy demand statistics. IEA [online]. Available at: https://www.iea.org/statistics/?country=WORLD\&year=2016\&category=Energy\%20supply\&indicato $r=$ TPESbySource \&mode=chart\&dataTable=BALANCES (Accessed 3 July 2019).

${ }^{2}$ de Vries, A. Joule 2(5), 801-805 (2018).

${ }^{3}$ Narayanan, A., Bonneau, J., Felten, E., Miller, A., and Goldfeder, S. Bitcoin and cryptocurrency technologies. (Princeton University Press, NJ., 2016)

${ }^{4}$ Mora, C. et al. Nature Climate Change 8, 931-933 (2018).

${ }^{5}$ Bendiksen, C., and Gibbons, S. The Bitcoin mining network: Trends, Average Creation Costs, Electricity Consumption \& Sources, CoinShares Research [online]. Available at:

https://coinshares.co.uk/assets/resources/Research/bitcoin-mining-network-june-2019-fidelityforeword.pdf (Accessed on 5 July 2019).

${ }^{6}$ Krause, M. J., and Tolaymat, T. Nature Sustainability 1(11), 711 (2018).

${ }^{7}$ Stoll, C., Klaaßen, L., and Gallersdörfer, U. Joule 3, 1-15 (2019).

${ }^{8}$ Howson, P., Oakes S., Baynham-Herd Z., and Swords J. Geoforum 100 (1), 1-9 (2019).

${ }^{9}$ Huckle, S., and White, M. Future Internet 8(4), 49-64 (2016).

${ }^{10}$ Gil-Pulger, J. Ethereum 2.0 PoS blockchain aims to cut energy use by $99 \%$. Bitcoinist [online]. Available at: https://bitcoinist.com/ethereum-pos-blockchain-cut-energy/ (Accessed 19 June 2019).

${ }^{11}$ Poseidon. (2019). Poseidon [online]. Available at: https://poseidon.eco/solution.html (Accessed 6 July 2019).

${ }^{12}$ Lang, C. Can buying Ben \& Jerry's ice cream save the Cordillera Azul National Park in Peru? REDDMonitor [online]. Available at: https://redd-monitor.org/2018/11/09/can-buying-ben-jerrys-icecream-save-the-cordillera-azul-national-park-in-peru-featuring-ecosphere-althelia-the-poseidonfoundation-redd-blockchain-and-the-government-of-malta/ (Accessed 19 June 2019).

${ }^{13}$ Enrici, A., and Hubacek, K. Ecology and Society 23(2), 7 (2018).

${ }^{14}$ [UNEP] United Nations Environment Programme. Carbon offsets are not our get-out-of-jail free card. UNEP [online]. Available at:

https://web.archive.org/web/20190610044930/https://www.unenvironment.org/news-andstories/story/carbon-offsets-are-not-our-get-out-jail-free-card (Accessed 4 July 2019).

${ }^{15}$ Regen.Network. Regen.Network Whitepaper [online]. Available at: https://www.regen.network/ (Accessed 3 June 2018).

${ }^{16}$ Howson, P. Conservation and Society 15(2), 125-135 (2017). 